\title{
Modular Verification of Vehicle Platooning with Respect to Decisions, Space and Time ${ }^{\star}$
}

\author{
Maryam Kamali, Sven Linker, and Michael Fisher \\ University of Liverpool, UK \\ \{maryam.kamali,s.linker,mfisher\}@liverpool.ac.uk
}

\begin{abstract}
The spread of autonomous systems into safety-critical areas has increased the demand for their formal verification, not only due to stronger certification requirements but also to public uncertainty over these new technologies. However, the complex nature of such systems, for example, the intricate combination of discrete and continuous aspects, ensures that whole system verification is often infeasible. This motivates the need for novel analysis approaches that modularise the problem, allowing us to restrict our analysis to one particular aspect of the system while abstracting away from others. For instance, while verifying the real-time properties of an autonomous system we might hide the details of the internal decision-making components. In this paper we describe verification of a range of properties across distinct dimesnions on a practical hybrid agent architecture. This allows us to verify the autonomous decision-making, real-time aspects, and spatial aspects of an autonomous vehicle platooning system. This modular approach also illustrates how both algorithmic and deductive verification techniques can be applied for the analysis of different system subcomponents.
\end{abstract}

Keywords: Modular Verification - Hybrid Agent Architecture $\cdot$ Automata $\cdot$ Spatial Reasoning · BDI Agent Programming

\section{Introduction}

Autonomous systems are increasingly being introduced into safety-critical areas, for example nuclear waste management [2], domestic robotics [5], or transportation, in the form of unmanned aircraft, advanced driver assistance systems, and even "driverless" cars. Although autonomous cars are generally aimed at increasing the overall safety of traffic, vehicle platooning [17|26], shown in Fig. 1] in particular provides even more advantages over single vehicles: it potentially decreases both congestion on motorways, and fuel consumption, since the relative braking distance between vehicles should be smaller, and hence the vehicles can make use of slipstreams with reduced wind resistance. Here, vehicles are held in sequence on a highway, with distances and speeds controlled by the platoon rather than the individual vehicle. Platooning has been recognised as a valuable means to achieve these goals, and is encouraged politically, for instance, by the Department of Transport of the United Kingdom 1

\footnotetext{
* Work supported EPSRC grants EP/N007565 (Science of Sensor Systems Software), EP/R026092 (FAIR-SPACE RAI Hub) and EP/L024845/1 (Verifiable Autonomy).

1 https://trl.co.uk/news/news/government-gives-green-light-firstoperational-vehicle-platooning-trial
} 
Autonomous vehicles within a platoon need to be verified to ensure the overall safety of the platoon. Specifically both autonomous decision-making concerning leaving/joining the platoon, and low-level interaction with its environment have to be analysed, in the best case by providing guarantees for reliable behaviour. To certify the highlevel decisions of an individual autonomous system, the rational agent concept [27] is widely used, since it allows for an analysis of the reasons why an autonomous system chooses a certain action.

The physical interaction of a vehicle with the rest of the platoon of vehicles in its environment consists of several different dimensions. Two of the most important are time and space. Timing constraints are of major importance to the overall behaviour of a system. For example, if an unsafe situation is encountered, the vehicles have to react within a certain time frame to ensure safety during emergencies. But even for normal vehicle behaviour, such as joining or leaving a platoon, time constraints are eminently important [8]. Spatial aspects are vital for similar reasons. Ensuring that vehicles do not get too close, or can fit in the space they are trying to move in to, is clearly important.

So now we reach the key problem. A complex, autonomous system such as an automotive vehicle platoon, will incorporate a diverse range of properties and behaviours. If we wish to formally verify all of these dimensions together then we will certainly hit complexity issues - multi-dimensional formalisation easily become very complex [7|14]. Two approaches are either to use modular verification techniques [19] or to use abstraction techniques [10] to separate out dimensions of concern.

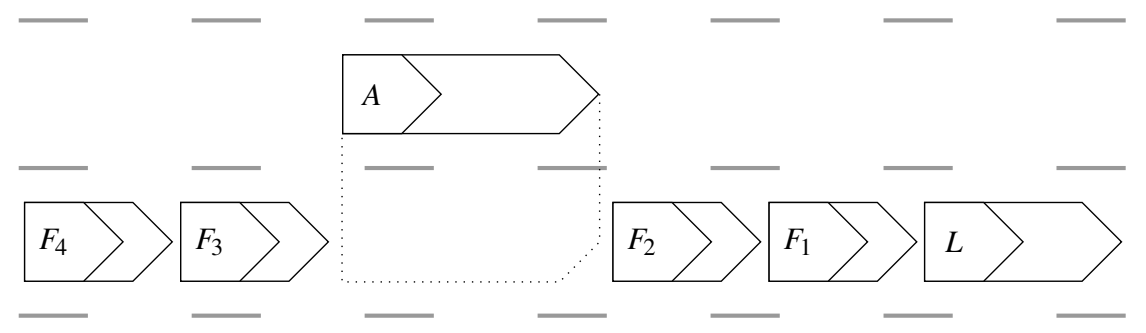

Fig. 1: Vehicle Platooning — vehicle $A$ joining interior of platoon

Our Approach. We have identified three key dimensions within autonomous vehicle platoons that we wish to assess: autonomous decision-making, real-time properties, and spatial properties. We also aim to minimise the change to existing components of the system when new components are introduced. Consequently, we use abstraction techniques for the three dimensions, but ensure that verification results for parts of the system that are unchanged remain valid, and so the verification task is reduced to checking any new system components. We show the applicability of this approach by taking an existing autonomous vehicle platoon system whose decision-making and real-time properties have already been verified, in [18], and incorporating spatial aspects. A spatial controller is introduced to model the lane-changing behaviour of the vehicles in the platoon. This was something that the original platoon verification from [18] did not consider and we now show that not only does the high-level decision making (agent) 
code remain unchanged, but since the spatial aspects were shown to be correct in [15], the new verification task is reduced to the analysis of the real-time requirements.

Consequently, we show how this modular verification approach supports the flexibility of the underlying hybrid agent architecture, with any new components of the extended architecture still being verifiable. The verification of such architectures remains feasible as long as we can apply appropriate abstraction to the system components.

\section{Hybrid Agent Architecture}

Cyber-physical systems, such as autonomous vehicles, require a sophisticated architecture together with corresponding formalism. Practical systems combine continuous environmental interactions, through feedback control, together with discrete changes between these control regimes. In traditional hybrid systems, separating the high-level decision making from continuous control concerns is difficult. The other drawback of standard hybrid modelling approaches is that the representation of decision-making can become very complex and hard to distinguish. We utilise a hybrid agent architecture [20] where the decision-making aspect is separated into a distinguished 'agent' while the system still provides for traditional feedback control systems. This approach to the modelling and development of autonomous systems provides a clear separation between these two concerns, and also the behaviour of each component is described in much more detail that can contribute to reason about their behaviours separately. Thus, the separation of high-level decision making and low-level controllers within a hybrid agent architecture provides an infrastructure for modular verification.

In this paper, we use a hybrid agent architecture, proposed for autonomous vehicle platooning in [18], as shown in Fig. 22 A Decision-Making Agent instructs a Physical and Continuous Engine by passing instructions through an Abstraction Agent. The Abstraction Agent receives streams of continuous data from the Physical and Continuous Engines, extracts discrete information from this, and sends it to the decision-making agent. The Physical and Continuous engine manages the real-time continuous control of the vehicle through feedback controllers, implemented in MATLAB. We assumed that the dynamics of the vehicles are continuous, i.e., they may not arbitrarily change positions and velocities. An automotive simulator, TORCS ${ }^{2}$ is used to implement the automotive environment and this environment is observed through the sensory input by the Physical and Continuous engine.

The Decision-Making Agent is a rational agent [27] that not only makes decisions, but will have explicit reasons for making these decisions. This allows us to describe what the autonomous system chooses to do, and to reason about why it makes its choices. Our Decision-Making Agent is based on the BDI (Belief-Desire-Intention) paradigm. Here, beliefs represent the agent's views about the world, desires provide the long-term objectives to be accomplished, and intentions capture the set of goals currently being undertaken by the agent in order to achieve its desires.

The separation between the Decision-Making Agent and the Physical and Continuous Engine provides a way to verify the agent behaviour in isolation from the detail of feedback control. In this work we utilise program model-checking over the DecisionMaking Agent. This allows us to formally verify the real agent code rather than a model

\footnotetext{
2 The Open Racing Car Simulator https://sourceforge.net/projects/torcs
} 


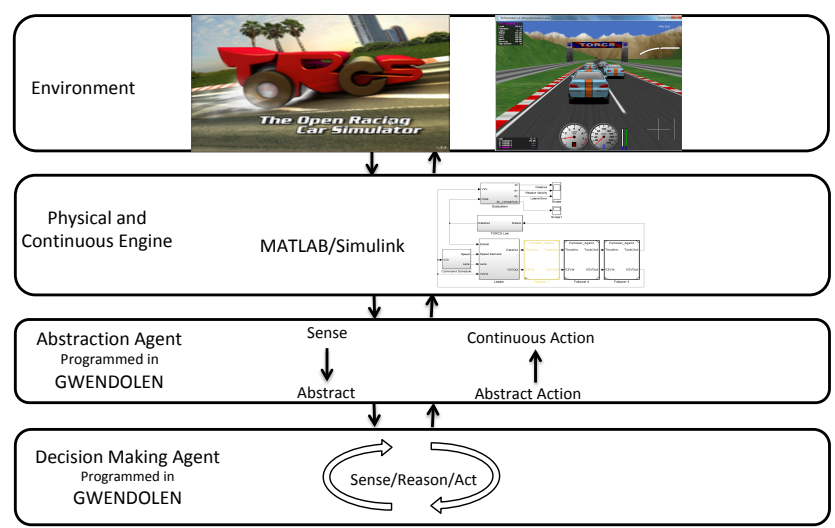

Fig. 2: Hybrid Agent Architecture [18]

of the agent behaviour. This formal verification of agent behaviour is carried out using the AJPF model checker and the agent itself is implemented in the verifiable language GWENDOLEN [11]. The model-checking approach using AJPF is used to demonstrate that the BDI agent always behaves according to the platoon requirements and never intentionally chooses unsafe options. Unfortunately, model checking of BDI agents through AJPF is not only resource-heavy, but also lacks support for the formal verification of timed behaviours. As indicated above, timing will be a key principle of relevance to safety-critical behaviour and so, to tackle this problem, Kamali et al. [18] proposed a modular approach to the verification of automotive platoons constructed in this way. They used a combination of AJPF, for internal agent decisions, and the Uppaal model checker, for global timing behaviours.

We here consider two of the main platooning procedures involved in joining and leaving a platoon. Both the joining and leaving procedures are comprised of a series of communications between an individual vehicle and the platoon leader aimed to obtain permission to join/leave or update the leader when the joining/leaving procedure is accomplished. Apart from the required communications, the vehicle switches between different controllers, such as moving from 'manual' to 'automatic' for speed and steering. One of the challenging manoeuvres is changing lanes and the high-level behaviour of the platoon is verified under the assumption that the lane changing manoeuvre is carried out safely. In order to accomplish the fully autonomous platooning while preserving safety, we extend the previous work of [18] by adding spatial reasoning to the platooning architecture. Representing space allows us to model the spatial controller of the system and consequently to verify the safety of the spatial controller behaviours.

Both the idea, and the concrete definition of the spatial controller, is taken from previous work [15]. The level of this spatial abstraction is still very high: we do not refer to specific/metric distances, but instead associate regions of space with different, abstract, properties. That is, we distinguish two different aspects of space needed by a vehicle: its reservation and its claim. The intuition here is that the reservation of a 
vehicle denotes the part of space that is necessary for the vehicle to operate safely. It comprises both the physical extent of the vehicle and the distance it needs to come to a standstill in case of an emergency. The claim, however, is not as restrictive. It is an additional way for the vehicles to communicate, similar to the turning signals common to road vehicles. That is, a vehicle sets a claim somewhere on the motorway to indicate its desire to occupy this part of the motorway in the (near) future. If the vehicle decides that changing to the new lane is safe, it mutates its existing claim into a reservation. Consequently, within our abstraction the vehicle is considered to be on both lanes at once, thus modelling the act of changing lanes. For example, in Fig. 1. the car $A$ currently set a claim on the right lane, to join the platoon.

\section{Methodology}

In this section, we show how the hybrid agent architecture of Sect. 2 can be instantiated to verify vehicle platooning with respect to the agent's decisions, the continuous behaviour, and the topological spatial changes necessary to change lanes, e.g., while joining a platoon. To that end, we refine the instantiation of previous work [18] with a new controller responsible for the spatial aspects of traffic, which in turn is inspired by previous work of one of the authors [15]. Generally, our system consists of several controllers, which constrain the possible behaviour of the vehicles on the road. This implies, in particular, that the behaviour of the parallel product of two components is a subset of the behaviour of each single component. To show the correctness of our refinement step, we prove a set of proof obligations including deadlock freedom and invariant preservation. We also show that all the verified properties of autonomous vehicle platooning presented in [18], hold after the refinement step.

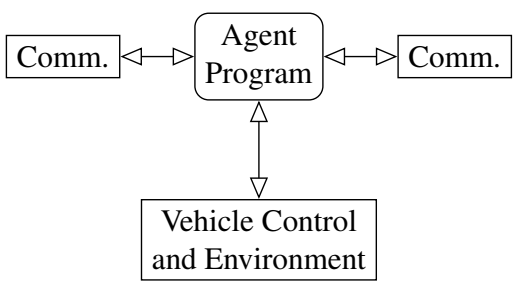

(a)

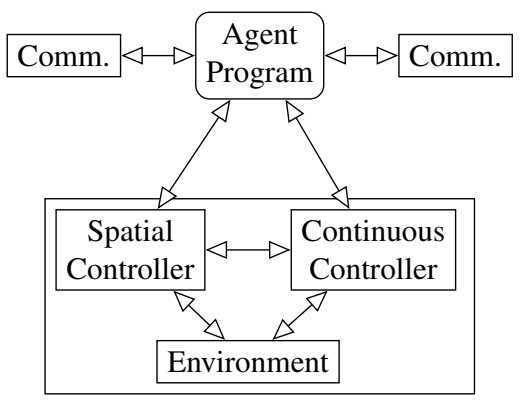

(b)

Fig. 3: Original and Refined Architecture

Fig. 3 shows both the original and refined architecture modelling an individual vehicle within a platoon. The centre of the architecture consists of the agent program, which makes autonomous decisions for the vehicle and may both communicate with other agents via some communication channel, and with both a continuous controller and an 
environment (cf. Fig. 3a). A main feature of our approach is a translation of the different components into simpler abstractions for verification purposes. That is, to verify the agent program, we can abstract from the timing aspects of the continuous controller. Thus we gain a simple (finite-state) timed automaton as the abstraction of the continuous behaviour. Similarly, we can reduce the agent program to the few parts necessary for the communication with the continuous controller for the verification of the latter. In both cases, the state space is reduced significantly, making verification feasible, in the case of the agent program by using AJPF [12] and in the case of the continuous controller by using UPPAAL [6].

\subsection{Agent}

The BDI agent program in our architecture is written in GWENDOLEN [11], a prologstyle programming language that incorporates explicit representation of goals, beliefs, and plans. AJPF is a model checker that accepts GWENDOLEN code as an input model. It allows for the specification and verification of agent properties with respect to beliefs and intentions. Since the general interface between the underlying vehicle implementation and the agent is similar to [18], we could re-use that agent program with only minor changes. We distinguish between two agent programs: the leader, which manages all joining and leaving requests of vehicles within, or outside, the platoon, and the follower, which defines the functionality of vehicles within the platoon.

We did not need to change the structure of the leader protocol, which is why we subsequently concentrate on the follower. The follower currently implements the interactions for four main features:

1. joining a platoon;

2. leaving a platoon;

3. switching the steering control between manual and automatic; and

4. setting a new distance to the front vehicle.

A vehicle intending to join to a platoon initially sends a joining request to the leader and waits for confirmation from the leader. When it receives the confirmation, it instructs the vehicle to change lane and waits for the vehicle to send back a successful confirmation of changing lane. After receiving the successful confirmation the follower switches its speed controller to automatic. When the joining vehicle is close enough to the proceeding follower within the platoon the agent instructs the vehicle to switch the steering controller to automatic. Finally, the joining vehicle confirms the successful joining procedure to the leader. When the joining vehicle receives a reply back from the leader, it deduces that the the joining goal has been achieved. The following code shows a simplified plan of the agent code for when the joining vehicle switches its speed controller from manual to automatic:

\footnotetext{
+ ! joining $(X, Y):$ (B name $(X), B$ join_agreement $(X, Y), B$ changed_lane, $\sim$ B speed_contr, $\sim$ B steering_contr, $\sim$ B joining_distance $<-+$ !speed_contr $(1)$, joining_distance ;
}

Here: + ! joining $(\mathrm{X}, \mathrm{Y})$ indicates the addition of the goal to join to the platoon; clauses within $\{\ldots\}$ states the conditions about the agent's beliefs which must be true 
such as a join agreement belief for joining behind the follower $Y$ (B join_agreement $(\mathrm{X}$, $\mathrm{Y})$ ); and + ! speed_contr $(1), *$ joining_distance , called the 'body' of a plan, is a set of deeds the agent performs for execution of the plan. + ! speed_contr(1) adds a new goal for switching the speed controller to automatic and $*$ joining_distance indicates that the execution of the plan is suspended until the joining vehicle reduces the gap with its immediate follower.

Given agent code, one can specify the agent properties with respect to beliefs, goals, and actions and then verify them using the AJPF model checker. As mentioned earlier, due to our modular verification approach we could skip the re-verification of previous platooning properties. An example of a safety property is as follows:

$$
\begin{aligned}
& \square\left(\mathrm{G}_{\mathrm{X}} \text { joining }(\mathrm{X}, \mathrm{Y}) \& \neg \mathrm{B} \text { X join_agreement }(\mathrm{X}, \mathrm{Y})\right) \\
& \left.\rightarrow \square \neg \mathrm{D} \mathrm{x} \text { perf( } \text { speed }_{\mathrm{C}} \text { ontroller }(1)\right)
\end{aligned}
$$

where $\mathrm{X}$ refers to a joining vehicle that has a goal to join to a platoon, in front of a platoon follower Y. $G_{\mathrm{X}}$ joining $(\mathrm{X}, \mathrm{Y})$ indicates a joining goal that agent $\mathrm{X}$ tries to achieve. $B_{X}$ join_agreement $(\mathrm{X}, \mathrm{Y})$ indicates the join agreement belief of agent $\mathrm{X}$, and $D_{X}$ perf(speed_controller(1)) indicates the action of perf(speed_controller(1)) that agent $X$ performs. This property denotes that if a vehicle never believes it has received a confirmation from the leader, then it never switches to the automatic speed controller.

\subsection{Continuous Controller}

In the original architecture, we combined the continuous controller and the environment into one entity. For example, we did not distinguish between interactions of the agent with the actuators of the autonomous system and interactions with the human driver. In both cases, the main feature of the interaction we were concerned with was the time taken for the controller or environment to react.

As shown in Fig. 3b, we now refine the continuous controller and the environment into three sub-components. We introduce two controllers, one referring to the timing aspects and the continuous behaviour of the vehicle, and the other specifically to control actuations with respect to space.

The refinement extends the previous environment with a model of potential collision, which will be defined in the subsequent section. It removes the nondeterministic failure of changing lane from the continuous controller that implicitly modelled the existence of such a potential collision. A part of the continuous controller automaton that has changed in our refinement step is shown in Fig. 4. Note that synchronisation channels are changed from changing_lane to phy_changing_lane since the refined continuous controller is synchronised with the spatial controller, while in the previous controller it was synchronised with the agent automaton. We elaborate on the spatial controller in the following section.

\subsection{Introducing Space}

In this section, we present the concrete instantiation of the spatial controller, as well as the translation into timed and untimed automata for verification purposes. To that end, 


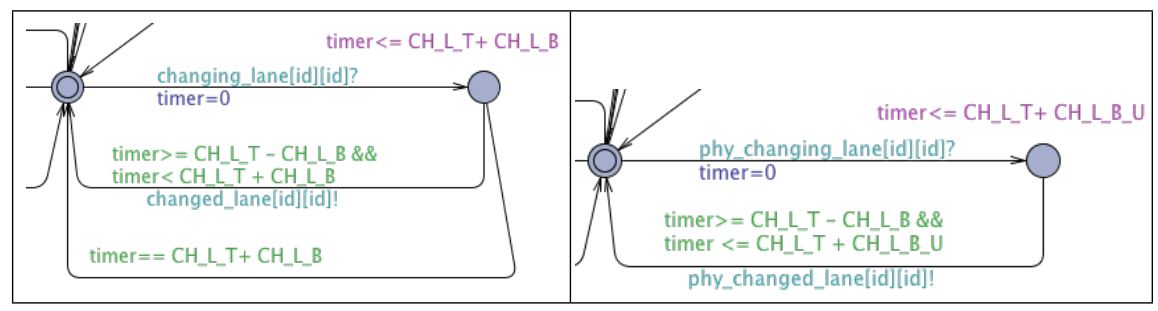

Fig. 4: Abstract and Refined Continuous Controller Automata

we formalise the ideas presented in Sect. 2 on the spatial model. However, we will not go into all of the details of the model of space, but refer to previous work [15]21].

We fix a set of lanes $\mathbb{L}=\{1, \ldots, n\}$ and for simplicity assume the motorway to be infinitely long. The dimension in the direction of the motorway, called the extension, is thus modelled by the real numbers $\mathbb{R}$. At any point in time, each vehicle $c$ is then spatially characterised by its position $\operatorname{pos}(c) \in \mathbb{R}$, its physical size $p s(c) \in \mathbb{R}$, its braking distance, i.e., the distance it needs to come to a standstill $b d(c) \in \mathbb{R}$, as well as the lanes it reserves $\operatorname{res}(c) \subseteq \mathbb{L}$ and claims $\operatorname{clm}(c) \subseteq \mathbb{L}$. These sets of lanes are subject to certain conditions (e.g., the set of claims has to be a singleton and has to be adjacent to the current reservation, etc.), which we will not expand upon Each vehicle $c$ can also perform certain actions, in particular

$$
\begin{aligned}
& c(c, n): \text { create a claim on lane } n \\
& r(c): \text { change an existing claim into a reservation } \\
& w d c(c): \text { remove/withdraw an existing claim } \\
& w d r(c, n): \text { shrink its reservation to only be on lane } n
\end{aligned}
$$

While the original defintion of the spatial model allowed for arbitrarily many, even infinite, of these instantaneous transitions at any point in time, we now restrict the possible transitions such that after each transition an amount of time greater than zero has to pass. We add this constraint to enforce the permanency of spatial changes on the road. Subsequently, we will refer to this model of space as $R$.

Using these abstract definitions as the semantics, we defined a specification logic with an emphasis on multi-lane traffic [15]. However, in this work we will not require the full logic, and hence we only explain the necessary details. One main feature of the logic is that it employs local reasoning, that is, a formula is evaluated with respect to a finite part of the motorway, as perceived by a distinguished vehicle, which is sometimes referred to as the ego vehicle. This finite perception is called the view of a vehicle, and consists of a subset of the lanes, as well as a finite interval of the real numbers, the extension of the view. We employ two spatial atoms $r e(c)$ and $c l(c)$, which denote that the current view consists of a single lane and a non-empty extension, and is fully occupied by the reservation (claim, respectively) of $c$. Furthermore, we use a single modality somewhere $\langle\varphi\rangle$, which denotes that the formula $\varphi$ holds somewhere on the space under consideration. With these specific definitions, and standard first-order operators, we can 


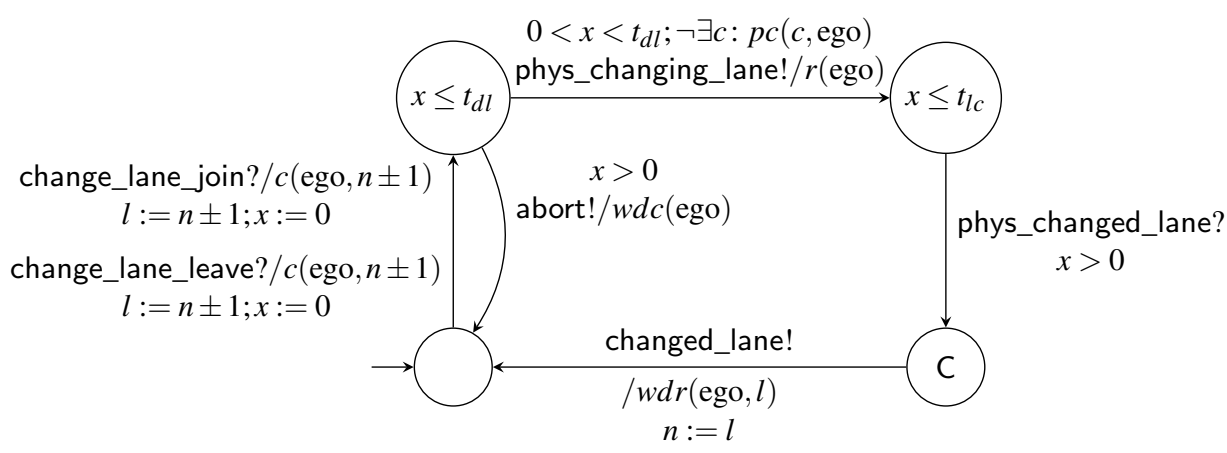

Fig. 5: Spatial Controller for Joining and Leaving a Platoon $\left(t_{d l}<t_{l c}\right)$

express the following two formulas.

$$
\begin{aligned}
c c & \equiv \neg \exists c: c \neq \operatorname{ego} \wedge\langle\operatorname{re}(\text { ego }) \wedge \operatorname{re}(c)\rangle \\
p c(c, \text { ego }) & \equiv c \neq \operatorname{ego} \wedge\langle c l(\text { ego }) \wedge(r e(c) \vee c l(c))\rangle
\end{aligned}
$$

Formula $c c$ denotes the existence of a vehicle $c$ whose reservation overlaps with the reservation of ego. According to our explanations above, this would amount to an unsafe situation. For simplicity, we term such situations as collisions, even though $c$ may only have encroached upon the braking distance of ego or vice versa. Formula $p c(c$, ego $)$ denotes that the claim of ego overlaps with either the claim of $c$ or its reservation. This may result in an unsafe situation, if ego changed its claim into a reservation. Hence, $p c(c$, ego $)$ allows us to identify potentially unsafe situations, and so take measures to mitigate this.

To model the spatial behaviour of a vehicle joining or leaving the platoon, we will use a type-amended timed automata called automotive-controlling timed automata (ACTA) [16]. These augment timed automata with the possibility to use spatial formulas as guards and invariants, as well as to use the spatial actions described above at the transitions. Figure 5 shows the controller in terms of an ACTA, where ego refers to the vehicle the controller is implemented in. The upper part of the controller is concerned with the vehicle trying to join a platoon, while the lower part handles the leaving of a platoon. The actions change_lane_join, change_lane_leave, changed_lane, and abort are used to communicate with the decision making agent. The first two actions are used by the agent to initiate the corresponding manoeuvre, while the spatial controller uses changed_lane and abort to indicate a successful and unsuccessful lane-change manoeuvre, respectively. The channel phys_changed_lane is a direct communication link with the continuous controller, which indicates that steering onto the new lane was successful. Observe that we chose to encode the necessary delays after the transitions into this controller as well via the clock $x$.

For the verification of the other components, we need to provide abstractions from the ACTA given above into both an untimed automaton, and a standard timed automaton. To abstract from both the timing and spatial definitions, we remove all references to clocks, spatial formulas and spatial actions, i.e., we only keep the discrete actions, 
and therefore maintain the order of actions. In this way, we create a simple finite automaton which serves as the abstraction of the spatial controller that can only be used during the verification of the agent programs. The translation into timed automata is slightly more involved. We employ a global set of identifiers for each vehicle. In fact, this set was already used to identify the different vehicles by parameterising the continuous controllers [18]. Hence, we replace each occurrence of ego with the parameter $i d$. Furthermore, we introduce a global array $c$ of Boolean values, where the identifiers serve as the indices, and each entry denotes whether the corresponding vehicle currently possesses a claim. Whether a vehicle is currently engaged in a lane-change manoeuvre, i.e., whether it uses two lanes at once for its reservation, is indicated by a variable $r$, which is local to each controller.

However, since claims and reservations are strongly tied together, we also need to define an abstraction of the road's behaviour. To that end, we chose to use a very simple abstraction: a potential collision can only happen, if at least one vehicle currently holds a claim. Furthermore, a potential collision has to last an arbitrary amount of time greater than zero before it can be resolved. This is a result of the assumption on the vehicles dynamics to be continuous and the necessary delays after the spatial transitions. Note that a potential collision can happen due to two reasons: either a spatial transition or the different velocities of two cars. In both cases, our model and its assumptions ensure that the situation persists for a non-zero amount of time. We can formalise these two properties with the following abstraction of the road's behaviour, as shown in Fig.6 In this figure, $y$ is a clock used to enforce the timing behaviour.

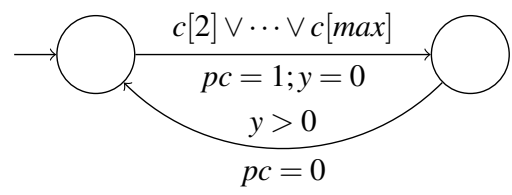

Fig. 6: Abstraction of Spatial Behaviour on the Road

With these changes, the timed abstraction of the spatial controller is as shown in Fig. 7. The timing behaviour is exactly as in the original automaton. Hence, if we can verify the other controllers in the presence of this controller, we can guarantee the safety of the overall system.

Finally, we need to define how the agent program and the continuous controller can be abstracted for the verification of the spatial properties. To that end, observe that the specification logic for the spatial properties does not contain modalities to refer to timings or decisions of the agent. That is, for the spatial properties, we do not refer to either time constraints or the specific goals or intentions of the agent. Hence, for spatial verification, we use the untimed automaton of the continuous controller which was also used during the verification of the agent. Similarly, the untimed abstraction automaton of the agent program used in the verification of timing aspects can be re-used during the verification of spatial properties. 


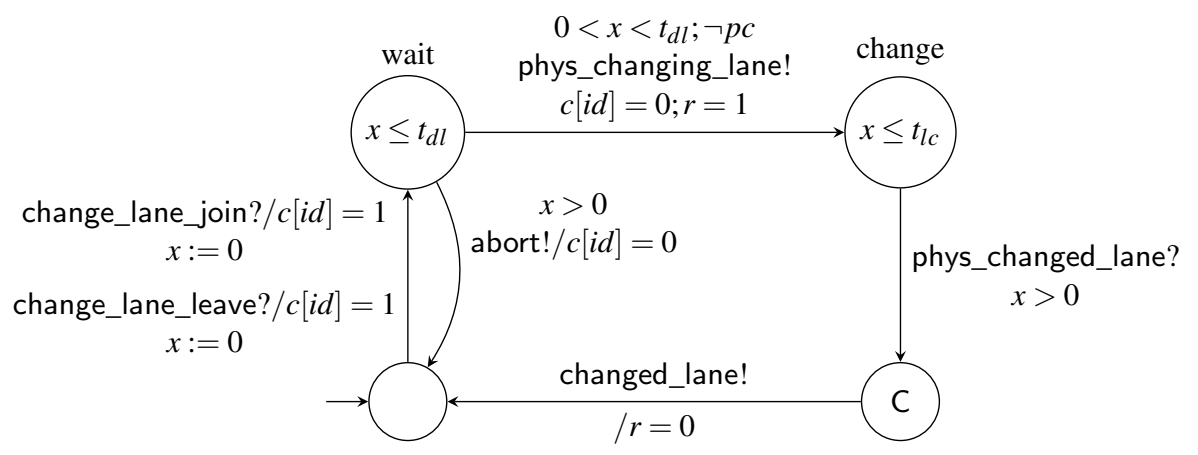

Fig. 7: Timed Abstraction of Spatial Controller of Fig.5 $5\left(t_{d l}<t_{l c}\right)$

Lemma 1. Let $A_{i}, V_{i}$ and $S_{i}$ be the agent program, continuous controller and spatial controller, respectively, of vehicle $i$, with $i \in\{1,2\}$. Furthermore let Comm 12 be the component modelling the communication of vehicle 1 and 2 . Let $S_{i}^{\prime}$ be the abstractions of the spatial controllers (Fig. 7), $A_{i}^{\prime}$ the abstractions of the agent programs, $R^{\prime}$ the abstraction of the road (Fig. 6), and $\varphi_{t}$ a formula describing a time property. If $A_{1}^{\prime}\left\|V_{1}\right\| S_{1}^{\prime} \|$ Comms $12\left\|A_{2}^{\prime}\right\| V_{2}\left\|S_{2}^{\prime}\right\| R^{\prime} \models \varphi_{t}$ then $A_{1}\left\|V_{1}\right\| S_{1} \|$ Comms $12\left\|A_{2}\right\| V_{2}\left\|S_{2}\right\| R \models \varphi_{t}$.

Proof (Sketch). The timing behaviour of $A_{i}$ and $A_{i}^{\prime}$ is the same (cf. [18]). Furthermore, the timing constraints on $S_{i}$ and $S_{i}^{\prime}$ are also the same. Now, after each spatial transition, in the original $S_{i}$, some time has to pass. In both $S_{i}$ and its abstraction $S_{i}^{\prime}$, every time a clock is reset the guards on the outgoing transitions of the target state $s$ require the automaton to stay in $s$ for some time. Finally, if the abstraction $R$ flags a potential collision, then the original system possesses a trace containing at least one claim for a vehicle. Let us assume this claim is of vehicle 2 . Then, all possible traces starting from this configuration are also possible in the abstraction $R$. Hence, whenever we can prove that the abstraction satisfies a timed formula $\varphi_{t}$, the original system also satisfies $\varphi_{t}$.

Lemma 2. Let $A_{i}, V_{i}$ and $S_{i}$ be the agent program, continuous controller and spatial controller, respectively, of vehicle $i$, with $i \in\{1,2\}$. Furthermore let Comm 12 be the component modelling the communication of vehicle 1 and 2 . Now let $A_{i}^{\prime}$ and $V_{i}^{\prime}$ be the abstractions without references to spatial properties as described above. Then, if $A_{1}^{\prime}\left\|V_{1}^{\prime}\right\| S_{1} \|$ Comms $12\left\|A_{2}^{\prime}\right\| V_{2}^{\prime}\left\|S_{2}\right\| R^{\prime} \models \varphi_{s}$ then $A_{1}\left\|V_{1}\right\| S_{1} \|$ Comms $12\left\|A_{2}\right\| V_{2}\left\|S_{2}\right\| R=\varphi_{s}$.

Proof (Sketch). Again, this holds since the abstractions $A_{i}^{\prime}$ and $V_{i}^{\prime}$ allow for more behaviour than the original automata. Furthermore, the spatial properties may neither refer to the internals of the agent program, nor to time aspects of the system.

\section{Verification of Vehicle Platooning}

In this section, we explain the verification approach built on the methodology presented in Sect. $3^{3}$ On one hand, we did not have to re-run most of our verification methods

${ }^{3}$ The model and the verified properties can be found at https://github.com/ VerifiableAutonomy/AgentPlatooning 
from our previous work, particularly running AJPF, since we only refined non-agent parts of the system. On the other hand, we needed to show that the refinement step was valid by proving proof obligations. In the following, we first identify a set of proof obligations that we proved to verify our refinement step. We then denote the spatial properties that we checked for our concrete vehicle platooning. We also point out those parts of the system that remained unchanged and consequently not re-verified. Finally, we prove that the spatial controller which is added to our concrete vehicle platooning is a safe fragment of the space model in [15].

\subsection{Proof Obligations}

The refinement step allows us to introduce more details about the spatial properties of vehicle platooning. However, we need to ensure that the new details do not violate the system invariants, and do not introduce deadlocks. The checking needs to be considered for both verification of agent and timing behaviours. The untimed abstraction of the spatial controller only allows the same set of sequences of interactions with the agent. This means that we did not change the structure of the agent programs themselves; neither the leader nor the follower, i.e., the refinement step is correct wrt. agent behaviour. To discuss the correctness of our refinement step wrt. temporal behaviour, we check four main proof obligations, shown in Fig. 8. The first three obligations are verified using the Uppaal model checker, followed by a discussion of the correctness of the fourth obligation. We instantiated the agent timed automata, spatial, and continuous controllers for a platoon of four vehicles and one leader. We choose an arbitrary vehicle, for example vehicle 2 , to denote our proof obligations and properties of interest, and described these with respect to this vehicle. Note that $a 2$ is the follower agent program as implemented in vehicle 2 and $s 2$ is the lane-change (spatial) controller of the same vehicle.

\begin{tabular}{|c|c|}
\hline Deadlock Freedom & $A \square$ not deadlock \\
\hline Possible to join and leave & $\begin{array}{l}E \diamond a 2 . j o i n \_c o m p l e t e d \\
E \diamond \text { a2.leave_completed }\end{array}$ \\
\hline Time bound for joining and leaving & $\begin{array}{l}A \square \text { a2.join_completed } \mathbf{i m p l y} \\
\left(\text { a2.process_time }>=50 \wedge a 2 . p r o c e s s \_t i m e<90\right) \\
A \square \text { a2.leave_completed } \mathbf{i m p l y} \\
\left(\text { a2.process_time }>=30 \wedge a 2 . p r o c e s s \_t i m e<50\right)\end{array}$ \\
\hline No new communication transaction & $\begin{array}{l}\text { changes were restricted to continuous and spatial } \\
\text { controllers }\end{array}$ \\
\hline
\end{tabular}

Fig. 8: Proof Obligations, with formalisation in timed temporal logic ${ }^{4}$

The first proof obligation that we verified was deadlock freedom. We showed that our refinement step was not too restrictive. The second proof obligation ensures that adding the spatial controller does not decrease the functionality of the platooning, and

\footnotetext{
${ }^{4} A=$ "all paths"; $E=$ "exist a path"; $\square=$ "Always"; $\diamond=$ "Eventually"
} 
we checked whether joining and leaving procedures can occur. In the previous Uppaal model of platooning, we assumed that change lane could happen in $20 \pm C H \_L \_B$ where $C H \_L \_B$ was reflecting the uncertainty of the changing lane. In our refinement, the lower bound remains the same, however, the upper bound splits to two waiting times for free space $t_{d l}$ (cf. Fig. 5) and the uncertainty of the changing lane $\left(C H \_L \_B-t_{d l}\right)$. Therefore, we could show that the time bound of joining and leaving remain the same as the previous model (The third proof obligation in Fig. 8).

In the refinement step, we defined two new channels representing the communication between the spatial controller and continuous controller, phy_changing_lane and phy_changed_lane. As these channels are not used in any other parts of the system, we can guarantee that no new communication transition is added to any other part of our model.

\subsection{Spatial Properties of Vehicle Platooning}

We can verify that if a vehicle requested a lane-change, i.e., the spatial controller reaches the wait state (cf. Fig. 7), and still perceives a potential collision after the waiting time $t_{d l}$, then the corresponding manoeuvre in the agent program will fail.

$$
\left(s 2 . w a i t \wedge p c \wedge s 2 . x==t_{l d}\right) \longrightarrow\left(\text { a2.failed_to_join } \vee a 2 . f a i l e d \_t o \_l e a v e\right)
$$

In this formula $\longrightarrow$ denotes the "leads-to" operator of Uppaal. Observe that we cannot identify whether the join manoeuvre or the leave manoeuvre failed, since the spatial controller acts similarly for both manoeuvres. Note that identification of the manoeuvre can be easily implemented by adding a flag to the spatial controller automaton. We can also show that, whenever the spatial controller chooses that a lane-change can be safely initiated, it does not perceive a potential collision on the road. Furthermore, as long as it stays in this state, no potential collision can arise.

$$
A \square \neg(\text { s2.change } \wedge p c)
$$

This property shows that the space on the road as formalised in Fig.6 is "well-behaved" within this abstraction, since a potential collision can only happen, if a vehicle possesses a claim. However, if the controller of vehicle 2 is in state change, it already changed its claim to a new reservation. The time needed to verify these properties was similar to the time needed for the proof obligations, which, compared to our previous attempt, changed negligibly.

\subsection{Spatial Safety Property}

The main property that the spatial controller must ensure is that the space used by two different vehicles is disjoint. That is, it has to ensure that the formula $c c$ as shown in Sect. 3.3 is an invariant of the system. To that end, we re-use a verification result [21] of a much more general controller specification encoded in the theorem prover Isabelle/HOL [24]. Safety in this work means that $\forall e$ : $\operatorname{safe}(e)$ is a global invariant, where $\operatorname{safe}(e)$ is defined as follows.

$$
\operatorname{safe}(e) \equiv \square \neg \exists c: c \neq e \wedge\langle r e(e) \wedge r e(c)\rangle
$$


Observe that the modality $\square$ is similar in intent to the box-modality used in the previous sections, in that it quantifies over arbitrary transition sequences, but it does not allow us to specify timing constraints. To prove this property to be an invariant, we need two main assumptions:

1. All vehicles keep their distance to the vehicles in their front and back

2. All vehicles adhere to a certain protocol for changing lanes with respect to the platoon under consideration

We do not elaborate on the first assumption. However, the second assumption is that, the vehicle must not mutate its claim into a reservation, in case of a potential collision during the phase where a claim is held. Formally, we have the following constraint, where $\square_{\mathrm{r}(d)}$ quantifies over the transition where the vehicle $d$ changes its claim into a reservation and $c$ ranges only over the vehicles within the platoon.

$$
\mathrm{LC} \equiv \forall d: \exists c: p c(c, d) \rightarrow \square_{\mathrm{r}(d)} \perp
$$

For simplicity, assume that the platoon under consideration consists of two vehicles as in Sect. 3 That is, the platoon $P$ consists of the following components.

$$
P \equiv A_{1}\left\|V_{1}\right\| S_{1} \| \text { Comms } 12\left\|A_{2}\right\| V_{2}\left\|S_{2}\right\| R
$$

Now, let $\llbracket S_{1} \rrbracket$ and $\llbracket S_{2} \rrbracket$ be the possible behaviours allowed by the controllers $S_{1}$ and $S_{2}$ as presented in Sect. 3.3. Since the only transition to change a claim into a reservation is guarded by the potential collision check, we have for $i \in\{1,2\}$,

$$
\llbracket S_{i} \rrbracket \cap\left\{\operatorname{tr} \mid \operatorname{tr} \models \exists c: p c(c, i) \wedge \nabla_{\mathrm{r}(i)} \top\right\}=\emptyset
$$

That is, since the behaviour of the parallel product of $S_{1}$ and $S_{2}$ is a subset of both $\llbracket S_{1} \rrbracket$ and $\llbracket S_{2} \rrbracket$, we also get

$$
\llbracket S_{1} \| S_{2} \rrbracket \cap\left\{t r \mid t r \models \exists c: p c(c, i) \wedge \nabla_{\mathrm{r}(i)} \top\right\}=\emptyset .
$$

Since the other controllers may only further restrict the possible behaviour of the platoon, we also have

$$
\llbracket P \rrbracket \cap\left\{t r \mid \operatorname{tr} \models \exists c: p c(c, i) \wedge \diamond_{\mathrm{r}(i)} \top\right\}=\emptyset .
$$

Due to our assumption on the behaviour of all other vehicles, we can infer that $\llbracket P \rrbracket$ does not contain any traces where other vehicles create a reservation during a potential collision. Hence, we can strengthen this property even further.

$$
\llbracket P \rrbracket \cap\left\{t r \mid \operatorname{tr}=\exists c, d: p c(c, d) \wedge \nabla_{\mathrm{r}(d)} \top\right\}=\emptyset,
$$

which in particular implies $\llbracket P \rrbracket \subseteq S$. This yields $\llbracket P \rrbracket \models \mathrm{LC}$, which has been shown to ensure that $P=\forall e$ : $\square \operatorname{safe}(e)$. Hence, our controller is a refinement of the general case, which was shown to be safe. 


\section{Concluding Remarks}

Contribution. We presented a verification technique for autonomous systems based on the use of a hybrid agent architecture. The decomposition of concerns inherent in this architecture allows us to define different aspects of the system within different formalisms, which are tied together by the communication structure of the system and its timing constraints. For each of the formalisms we defined a translation into an abstraction compatible with the other formalisms. In this way, we can concentrate on each aspect in turn during verification, which both reduces the state space, and allows us to use different techniques for each aspect.

Decomposition techniques often isolate the single components and replace the interaction with the other components by assumptions, which are then shown to be guaranteed by the respective components [22]. In contrast, during each step of the verification, we keep the general structure of the overall system. That is, we do not really decompose the system, but abstract from different parts during each step. This eliminates the need to infer the behaviour of the single components, e.g., in the form of guarantees. Of course, this also means that large parts of the state space are retained during verification, in comparison to techniques which replace the other components with the guarantees they keep. However, we have shown that our approach is both feasible for autonomous systems, as well as that it scales well if new aspects are to be verified.

Related Work Müller et al. presented a technique to verify safety of hybrid systems [23] based on the identification of components. In their approach, they need to define and verify contracts for the behaviour of each component, which may simply assumed to be true during the verification of other components. In this manner, they can reduce the verification task for each component. Their systems need to be defined within a single formalism, differential dynamic logic [25], and are verified with the distinguished tool KeymaeraX [13]. In contrast, we can rather easily incorporate new formalisms into our approach, as evidenced by the introduction of the lane-change controller and the necessary spatial formalism. This is due to the minimised interaction between our controllers, in the form of time and communication. In this way, we may use the verification techniques suitable for the corresponding subsystems, as long as we have a sensible abstraction and refinement results for each system.

Abstraction and refinement techniques are often employed for verification purposes. For example, in counter-example guided abstraction (CEGAR) [9], the verification starts with a very broad abstraction. If a counter-example is found to be spurious, i.e., it is not viable in the original system, the corresponding part of the state-space has to be refined to eliminate this example. That is, the system is analysed in a top-down fashion, from the broadest possible abstraction to an explicit description of the system. Our method proceeds in a somewhat orthogonal way. Instead of building an abstraction of the system as a whole, we build several abstractions according to the type of property we intend to verify.

The concept of abstraction and refinement relations is used prominently in Event-B [1]. Banach and Butler have used a hybrid extension of Event-B to model and verify controllers used in autonomous driving [34]. However, these controllers have been verified on their own, and the interactions between them have not been verified. 


\section{References}

1. Abrial, J.R.: Modeling in Event-B: System and Software Engineering. CUP (2010)

2. Aitken, J., Shaukat, A., Cucco, E., Dennis, L., Veres, S., Gao, Y., Fisher, M., Kuo, J., Robinson, T., Mort, P.: Autonomous Nuclear Waste Management. IEEE Int. Sys. (2018)

3. Banach, R., Butler, M.: Cruise Control in Hybrid Event-B. In: Proc. International Colloquium on Theoretical Aspects of Computing. LNCS, vol. 8049, pp. 76-93. Springer (2013)

4. Banach, R., Butler, M.: A Hybrid Event-B Study of Lane Centering. In: Proc. International Conference on Complex Systems Design \& Management. pp. 97-111. Springer (2014)

5. Beer, J.M., et al: The Domesticated Robot: Design Guidelines for Assisting Older Adults to Age in Place. In: Proc. IEEE HRI. pp. 335-342 (2012)

6. Behrmann, G., et al: Uppaal 4.0. In: Proc. Int. Conf. Quantitative Evaluation of Systems. pp. 125-126 (2006)

7. Blackburn, P., van Benthem, J., Wolter, F. (eds.): Handbook of Modal Logic. Elsevier (2006)

8. Burns, A.: How to Verify a Safe Real-Time System: Application of Model Checking and Timed Automata to a Production Cell Case Study. Real-Time Systems 24(2), 135-151 (2003)

9. Clarke, E., Grumberg, O., Jha, S., Lu, Y., Veith, H.: Counterexample-Guided Abstraction Refinement. In: Proc. CAV. pp. 154-169. Springer (2000)

10. Clarke, E.M., Grumberg, O., Long, D.E.: Model Checking and Abstraction. ACM Trans. Program. Lang. Syst. 16(5), 1512-1542 (1994),

11. Dennis, L.A., Farwer, B.: Gwendolen: a BDI Language for Verifiable Agents. In: Proc. AISB 2008 Symp. Logic and the Simulation of Interaction and Reasoning. pp. 16-23 (2008)

12. Dennis, L.A., Fisher, M., Webster, M.P., Bordini, R.H.: Model Checking Agent Programming Languages. Automated Software Engineering 19(1), 5-63 (2012),

13. Fulton, N., Mitsch, S., Quesel, J.D., Völp, M., Platzer, A.: KeYmaera X: An Axiomatic Tactical Theorem Prover for Hybrid Systems. In: Proc. CADE. pp. 527-538. Springer (2015)

14. Gabbay, D., Kurucz, A., Wolter, F., Zakharyaschev, M.: Many-Dimensional Modal Logics: Theory and Applications. Elsevier Science (2003)

15. Hilscher, M., Linker, S., Olderog, E.R., Ravn, A.P.: An Abstract Model for Proving Safety of Multi-lane Traffic Manoeuvres. In: Proc. Formal Methods and Software Engineering. pp. 404-419. Springer (2011)

16. Hilscher, M., Schwammberger, M.: An Abstract Model for Proving Safety of Autonomous Urban Traffic. In: Proc. ICTAC. pp. 274-292. Springer (2016)

17. Hsu, A., Eskafi, F., Sachs, S., Varaija, P.: Protocol Design for an Automated Highway System. Discrete Event Dynamic Systems 2(1), 183-206 (1994)

18. Kamali, M., Dennis, L.A., McAree, O., Fisher, M., Veres, S.M.: Formal Verification of Autonomous Vehicle Platooning. Science of Computer Programming 148, 88-106 (2017),

19. Konur, S., Fisher, M., Schewe, S.: Combined Model Checking for Temporal, Probabilistic, and Real-time Logics. Theoretical Computer Science 503, 61-88 (2013)

20. Lincoln, N., et al: An Agent Based Framework for Adaptive Control and Decision Making of Autonomous Vehicles. In: Proc. IFAC Workshop on Adaptation and Learning in Control and Signal Processing (ALCOSP) (2010)

21. Linker, S.: Spatial Reasoning About Motorway Traffic Safety with Isabelle/HOL. In: Integrated Formal Methods. pp. 34-49. LNCS, Springer (2017)

22. Misra, J., Chandy, K.M.: Proofs of Networks of Processes. IEEE Trans. Software Engineering SE-7(4), 417-426 (1981)

23. Müller, A., Mitsch, S., Retschitzegger, W., Schwinger, W., Platzer, A.: A Component-Based Approach to Hybrid Systems Safety Verification. In: Proc. Integrated Formal Methods. pp. 441-456. Springer (2016) 
24. Nipkow, T., Paulson, L.C., Wenzel, M.: Isabelle/HOL - A Proof Assistant for Higher-Order Logic, LNCS, vol. 2283. Springer (2002)

25. Platzer, A.: Logical Analysis of Hybrid Systems: Proving Theorems for Complex Dynamics. Springer (2010)

26. Solyom, S., Coelingh, E.: Performance Limitations in Vehicle Platoon Control. IEEE Intell. Transport. Syst. Mag. 5(4), 112-120 (2013),

27. Wooldridge, M.J.: Reasoning about Rational Agents. MIT press (2000) 